\title{
Cellular Adaptation to Ischemia; Ischemic Conditioning to Confer Neuroprotective Benefits in Mild Cognitive Impairment and Alzheimer's disease
}

\author{
Nicole Hess* \\ School of Psychology, University of New England, Armidale, NSW 2350, Australia \\ ${ }^{\star}$ Correspondence to: Nicole Hess, School of Psychology, University of New England, Armidale, NSW 2350, Australia; E-mail: nhess2@une.edu.au
}

Received: June 06, 2019; Accepted: June 12, 2019; Published: June 20, 2019;

\begin{abstract}
Small, controlled doses of ischemia induced in a healthy limb has been demonstrated to strengthen the body's tolerance to larger more toxic doses. Ischemic conditioning utilising protocols of remote ischemic conditioning (RIC) and physiological ischemic training (PIT) trigger mechanisms of cellular adaptation to ischemia. These protocols may represent practical and translatable therapies for neurological diseases, such as mild cognitive impairment and Alzheimer's disease, that have an ischemic or inflammatory basis. Whilst the current literature supports the neuroprotective and anti-hypertensive effects of RIC and PIT, to date there has been no investigation into the effects of PIT utilising isometric exercise training (IET) on cognitive performance outcomes in an elderly neuropathological cohort. However, it seems feasible that the anti-hypertensive effects elicited through IET might be a stimulus for improvements in systemic and neurovascular circulation and as a result, enhanced cognitive performance.
\end{abstract}

Keywords: Alzheimer's Disease, Blood Pressure, Hypertension, Ischemic Conditioning, Isometric Exercise Training, Mild Cognitive Impairment, Reactive Hyperaemia Remote Ischemic Conditioning, Vascular Risk Factors

Ischemia is thought to play a pivotal role in the aetiology and progression of Alzheimer's disease [1-3] with reduced cerebral blood flow $(\mathrm{CBF})$ the manifestation of this [4]. Ischemia arises as a consequence of restricted blood flow and compromises the efficient circulation of oxygen, nutrients, other important blood borne factors, and the removal of toxic metabolic waste. Cellular dysfunction arising from ischemia will often result in cell death. Ischemia may be partial as in the case of hypoperfusion which occurs in conditions such as mild cognitive impairment $(\mathrm{MCI})$ and $\mathrm{AD}$ or total as in the case of heart attack and stroke. Ischemia is associated with serious physiological implications. However, through a process of ischemic conditioning, small controlled doses of ischemia delivered to a healthy limb is able to strengthen the body's tolerance to larger more toxic doses [5]. This type of treatment is currently administered to patients to improve the outcomes of heart attack [6] and ischemic stroke [7, 8]. Two methods of inducing remote limb ischemia are remote ischemic conditioning (RIC) and physiological ischemic training (PIT). RIC involves inflating a blood pressure cuff to above systolic blood pressure (SBP) to induce total ischemia via full occlusion of the brachial artery, followed by reperfusion after cuff deflation [9]. PIT involves subjecting skeletal muscle to intense contraction via the application of isometric contraction using handgrip dynamometer or tourniquet to induce partial occlusion of the brachial artery followed by reperfusion [10].

\section{Ischemic Conditioning}

The practice of ischemic conditioning to elicit cellular adaptation in the myocardium has been utilised for approximately 30 years [6].
Research investigating the neuroprotective potential of RIC began approximately10 years later with initial findings reporting RIC as an effective method of limiting neural tissue damage after stroke [11]. Since then there has only been a small amount of research in this area, with exciting results, Mouse models of vascular cognitive impairment have shown increased CBF, reduction in inflammation, reduced amyloid-beta deposition and improved cognition [12]. Human trials have shown increased CBF [9], the enhancement of neuroplasticity [13], and motor and cognitive learning enhancements in healthy adults [14].

As with RIC, PIT has been shown to stimulate collateral formation in the myocardium [15], upregulate vascular endothelial growth factor (VEGF) production, and promote angiogenesis [16]. More recent research reported that the application of PIT using isometric exercise training (IET), at 50\% Maximal Voluntary Contraction (MVC), to patients with acute cerebral infarction promoted brain collateral formation via the increased expression of VEGF and recruitment of endothelial progenitor cells [17]. The same researchers also reported a positive correlation between increased $\mathrm{CBF}$ and improved motor function. More recently, improvement in cognitive performance and reduction in systemic arterial stiffness was demonstrated in a cohort of non-elderly, non-neuropathological adults after 8 weeks of IET at $30 \%$ MVC [18]. Traditionally, IET using handgrip dynamometer has been successfully demonstrated as an anti-hypertensive therapy [19]. IET can elicit blood pressure (BP) reductions greater than those achieved through aerobic exercise and equivalent to those achieved 
through monotherapy with beta-blocker [20]. Furthermore, there is a considerable amount of research that links hypertension to conditions of cognitive decline such MCI and $\mathrm{AD}$ [21].

\section{The Role of Hypertension}

Hypertension, a hallmark of aging, has been linked with cerebrovascular pathology, hypoperfusion [22], and cognitive decline [23]. Imaging studies have demonstrated an association between brain atrophy and untreated hypertension [24] and positive correlations have been demonstrated among SBP, diastolic blood pressure, and burden of neural $\mathrm{AD}$ pathology $[21,25]$. Hypertension is associated with structural and functional changes in cerebrovascular pathways. These deleterious alterations are potentially reversible [26]. Subsequently, improving $\mathrm{CBF}$ may also lead to improvements in cognitive performance. The treatment and management of hypertension has been observed to slow cognitive decline in individuals with AD [27] and to reduce the risk of progression from $\mathrm{MCI}$ to $\mathrm{AD} \mathrm{Li} \mathrm{et} \mathrm{al.} \mathrm{[17].}$

\section{Change Mechanisms of Ischemic Training}

Whilst the signalling mechanisms involved in ischemic conditioning are not fully understood, evidence obtained through animal models and clinical trials suggest that signalling initiation occurs via autacoids such as adenosine, bradykinin, and calcitonin gene-related peptide. To varying extents each of these autacoids are involved in neuromodulation, inflammatory mediation, and vasodilation [28]. Signal transmission to the brain is believed to occur through an interaction between humoral and neural pathways such as peripheral and autonomic nervous systems, blood borne factors (IL-10, and nitrite), immune and anti-inflammatory factors (IL-6, IL10, IL-1ra), and endogenous carbon monoxide [9]. Abnormalities in the metabolism of endogenous carbon monoxide have been linked to a variety of diseases including neurodegenerations, hypertension, heart failure, and inflammation [29]. Mitochondria play a prominent role in signal transduction with most pathways that are triggered by ischemic conditioning converging on the mitochondria [5].. Mitochondrion are the energy packages of the cell and are responsible for regulating cell metabolism. Nitrite protects mitochondria from oxidative stress and is upregulated after the application of RIC, as is $\mathrm{CBF}$ [9]. Inadequate $\mathrm{CBF}$ is a contributor to oxidative stress in brain cells and has significant implications in neurological diseases such as $\mathrm{AD}$ where the mitochondria are ravaged by oxidative stress $[30,4]$.

\section{Considerations for Future Investigation}

Dementia is Australia's second leading cause of death in adults over 65 years [31] and is the greatest cause of disability among the elderly. Both RIC and PIT/IET represent practical and translatable therapies for neurological diseases that have an ischemic or inflammatory basis. Whilst the current literature supports the neuroprotective and antihypertensive effects of PIT/IET, to date there has been no investigation into the effects of this protocol on cognitive performance outcomes in an elderly neuropathological cohort. However, it seems feasible that the anti-hypertensive effects elicited through IET might be a stimulus for improvements in systemic and neurovascular circulation and as a result, enhanced cognitive performance.
The potential for limb ischemia to trigger neuroprotective physiological responses to support and repair the brain has been demonstrated via RIC and PIT/IET protocols and introduces exciting therapeutic potential for individuals with MCI and AD. The application of RIC and PIT/IET may create an ischemic event that is adequate to confer neuroprotective benefits and anti-hypertensive effects in elderly adults with cognitive impairment or AD. Further investigations within this domain have the potential to yield lifechanging results for many individuals.

\section{References}

1. de la Torre JC (2002) Alzheimer disease as a vascular disorder nosological evidence. Stroke 33: 1152-1162. [crossref]

2. de la Torre, J C (2010) Vascular risk factor detection and control may prevent Alzheimer's disease. Ageing Res Rev 9: 218-225. [crossref]

3. de la Torre JC, Mussivand T (1993) Can disturbed brain microcirculation cause Alzheimer's disease? Neurol Res 15: 146. [crossref]

4. Zlokovic BV (2011) Neurovascular pathways to neurodegeneration in Alzheimer's disease and other disorders. Nat Rev Neurosci 12: 723-738. [crossref]

5. Hess DC, Blauenfeldt RA, Andersen G, Hougaard KD, Hoda MN, et al. (2015) Remote ischaemic conditioning [mdash] a new paradigm of self-protection in the brain. Nat Rev Neurol 11: 698-710. [crossref]

6. Murry CE, Jennings RB, Reimer KA (1986) Preconditioning with ischemia: a delay of lethal cell injury in ischemic myocardium. Circulation 74: 1124-1136. [crossref]

7. Hougaard KD, Hjort N, Zeidler D, Sørensen L, Nørgaard A,et al. (2014) Remote Ischemic Perconditioning as an Adjunct Therapy to Thrombolysis in Patients With Acute Ischemic Stroke A Randomized Trial. Stroke 45: 159-167. [crossref]

8. Meng R, Ding Y, Asmaro K, Brogan D, Meng L, et al. (2015) Ischemic conditioning is safe and effective for octo-and nonagenarians in stroke prevention and treatment. Neurotherapeutics 12: 667-677. [crossref]

9. Hess DC, Hoda MN, Khan MB (2016) Humoral mediators of remote ischemic conditioning: important role of eNOS/NO/nitrite. Acta Neurochir Suppl 121: 4548. [crossref]

10. Ni J, Lu H, Lu X, Jiang M, Peng Q, et al. (2015) The evolving concept of physiological ischemia training vs ischemia preconditioning. J Biomed Res 29: 445-450. [crossref]

11. Kitagawa K, Matsumoto M, Tagaya M, Hata R, Ueda H, et al. (1990) 'Ischemic tolerance'phenomenon found in the brain. Brain Res 528: 21-24. [crossref]

12. Bink DI, Ritz K, Aronica E, van der Weerd L, Daemen MJ (2013) Mouse models to study the effect of cardiovascular risk factors on brain structure and cognition. $J$ Cereb Blood Flow Metab 33: 1666-1684. [crossref]

13. Cherry-Allen KM, Gidday JM, Lee JM, Hershey T, Lang CE (2015) Remote limb ischemic conditioning enhances motor learning in healthy humans. J Neurophysiol 113: 3708-3719. [crossref]

14. Cherry-Allen KM, Gidday JM2, Lee JM, Hershey T, Lang CE (2017) Remote limb ischemic conditioning at two cuff inflation pressures yields learning enhancements in healthy adults. $J$ Mot Behav 49: 337-348. [crossref]

15. Lin S, Lu X, Chen S, Ye F, Zhang J, et al. (2012) Human coronary collateral recruitment is facilitated by isometric exercise during acute coronary occlusion. $J$ Rehabil Med 44: 691-695. [crossref]

16. Zhao Y, Li J, Lin A, Xiao M, Xiao B, et al. (2011) Improving angiogenesis and muscle performance in the ischemic limb model by physiological ischemic training in rabbits. Am J Phys Med Rehabil 90: 1020-1029. [crossref]

17. Wan C, Li J, Yi L (2011) Enhancement of homing capability of endothelial progenitor cells to ischaemic myocardium through physiological ischaemia training. J Rehabil Med 43: 550-555. [crossref]

18. Dempster KS, McGowan CL, Wade TJ, O'Leary DD (2018) Effects of Isometric Handgrip Exercise Training on Systemic Arterial Stiffness, Cardiovagal Baroreflex Sensitivity, and Cognition in Treated Adults with Hypertension: A Pilot Study. Critical Reviews ${ }^{\mathrm{TM}}$ in Physical and Rehabilitation Medicine 30.

19. Carlson DJ, Dieberg G, Hess NC, Millar PJ, Smart NA (2014) Isometric exercise training for blood pressure management: a systematic review and meta-analysis. Mayo Clin Proc 89: 327-334. [crossref]

20. Wong GW, Wright JM (2014) Blood pressure lowering efficacy of nonselective beta-blockers for primary hypertension. Cochrane Database Syst Rev. 28: CD007452 [crossref]

21. Hess NC, Smart NA (2017) Isometric exercise training for managing vascular risk factors in mild cognitive impairment and Alzheimer's disease. Front Aging Neurosci 9: 48. [crossref] 
Nicole Hess (2019) Cellular Adaptation to Ischemia; Ischemic Conditioning to Confer Neuroprotective Benefits in Mild Cognitive Impairment and Alzheimer's disease

22. Brown, $\mathrm{W}$, Thore $\mathrm{C}$ (2011) Review: cerebral microvascular pathology in ageing and neurodegeneration Neuropathol Appl Neurobiol 37: 56-74. [crossref]

23. Reitz C, Tang MX, Manly J, Mayeux R, Luchsinger JA (2007) Hypertension and the risk of mild cognitive impairment. Arch Neurol 64: 1734-1740 [crossref]

24. Launer LJ, Masaki K, Petrovitch H, Foley D, Havlik RJ (1995) The association between midlife blood pressure levels and late-life cognitive function. JAMA 274: 1846-1851. [crossref]

25. Korf ES, White LR, Scheltens P, Launer LJ (2004) Midlife Blood Pressure and the Risk of Hippocampal Atrophy The Honolulu Asia Aging Study. Hypertension 44: 29-34. [crossref]

26. Lipsitz LA, Gagnon M, Vyas M, Iloputaife I, Kiely DK, et al. (2005) Antihypertensive therapy increases cerebral blood flow and carotid distensibility in hypertensive elderly subjects. Hypertension 45: 216-221. [crossref]

27. Deschaintre Y, Richard F, Leys D, Pasquier F (2009) Treatment of vascular risk factors is associated with slower decline in Alzheimer disease. Neurology 73: 674680. [crossref]
28. Steensrud T, Li J, Dai X, Manlhiot C, Kharbanda RK, Tropak M, et al. (2010) Pretreatment with the nitric oxide donor SNAP or nerve transection blocks humoral preconditioning by remote limb ischemia or intra-arterial adenosine. Am J Physiol Heart Circ Physiol 299: H1598-H1603. [crossref]

29. Wu L, Wang R (2005) Carbon Monoxide: Endogenous Production, Physiological Functions, and Pharmacological Applications. Pharmacol Rev 57: 585-630. [crossref]

30. Iadecola C, Park L, Capone C (2009) Threats to the mind aging, amyloid, and hypertension Stroke 40: 40-44. [crossref]

31. Australian Bureau of Statistics (2017) 33030 - Causes of death, Australia, 2016: Australian Bureau of Statistics Canberra, Australia.

\section{Citation:}

Nicole Hess (2019) Cellular Adaptation to Ischemia; Ischemic Conditioning to Confer Neuroprotective Benefits in Mild Cognitive Impairment and Alzheimer's disease. Ageing Sci Ment Health Stud Volume 3(2): 1-3. 